\title{
SHADOWING PROPERTY FOR INVERSE LIMIT SPACES
}

\author{
LIANG CHEN AND SHIHAI LI
}

(Communicated by Kenneth R. Meyer)

\begin{abstract}
We investigate the relationship between the shadowing property for continuous maps on a compact metric space and that for the shift maps on the inverse limit spaces. As an example, we show that the shift map of some pseudoarc has the shadowing property.
\end{abstract}

\section{Definitions AND THE MAIN RESUlT}

Let $(X, d)$ be a compact metric space and $f: X \rightarrow X$ be continuous.

The inverse limit space $\langle X ; f ; \underline{d}\rangle$ of $f$ is a metric space defined by the sequence

$$
X \stackrel{f}{\longleftarrow} X \stackrel{f}{\longleftarrow} X \stackrel{f}{\longleftarrow} \ldots
$$

whose elements $\underline{x}=\left(x_{0}, x_{1}, x_{2}, \ldots\right)$ satisfy $f\left(x_{i+1}\right)=x_{i}$ and the metric is defined by

$$
\underline{d}(\underline{x}, \underline{y})=\sum_{i=0}^{\infty} \frac{d\left(x_{i}, y_{i}\right)}{2^{i}} .
$$

Let $\langle X ; f\rangle$ denote the underlying set of $\langle X ; f ; \underline{d}\rangle$. The shift map $\sigma_{f}:\langle X ; f\rangle$ $\rightarrow\langle X ; f\rangle$ is defined by $\sigma_{f}\left[\left(x_{0}, x_{1}, \ldots\right)\right]=\left(f\left(x_{0}\right), x_{0}, x_{1}, \ldots\right)$ and the $i$ th projection $\pi_{i}$ of $\langle X ; f\rangle$ is defined by $\pi_{i}\left[\left(x_{0}, x_{1}, \ldots\right)\right]=x_{i}$ for each $i=$ $0,1, \ldots$ The metric $\underline{d}$ induces a topology that is equivalent to the relative product topology of $\prod_{i=0}^{\infty} X_{i}$ with $X_{i}=X$ restricted on $\langle X ; f\rangle$. A general discussion on the dynamical properties of the inverse limit spaces is contained in $[15,17]$.

Given $\delta>0$, a sequence $x_{0}, \ldots, x_{n}, \ldots$ of points in $X$ is a $\delta$-pseudo-orbit of $f$ if

$$
d\left(x_{n+1}, f\left(x_{n}\right)\right) \leq \delta \text { for every } n=0,1, \ldots
$$

For a given positive number $\varepsilon>0$, a sequence $\left\{x_{0}, \ldots, x_{n}, \ldots\right\}$ of points in $X$ is $\varepsilon$-shadowed under $f$ provided that there exists $y \in X$ satisfying

$$
d\left(x_{n}, f^{n}(y)\right) \leq \varepsilon \quad \text { for all } n \geq 0 .
$$

The shadowing property of $f$ on $(X, d)$ means that for any $\varepsilon>0$ there exists $\delta>0$ such that every $\delta$-pseudo-orbit is $\varepsilon$-shadowed. The earlier work

Received by the editors December 6, 1990.

1991 Mathematics Subject Classification. Primary 58F08; Secondary 26A18.

Key words and phrases. Shadowing property, inverse limit space, shift map, pseudoarc. 
on the shadowing property for diffeomorphisms and continuous maps includes [1-11, 13-14, and 16].

Here we introduce a new concept.

Definition 1.1. $f$ has the asymptotically shadowing property if for every $\varepsilon>0$ there exist a real number $\delta>0$ and an integer $N \geq 0$ such that for any $\delta$-pseudo-orbit $\left\{x_{0}, \ldots, x_{n}, \ldots\right\},\left\{x_{N}, x_{N+1}, \ldots\right\}$ is $\varepsilon$-shadowed.

By the definition, the shadowing property of $f$ implies the asymptotically shadowing property of $f$, but the converse is not ture (see Example 4.2). It is clear that the asymptotically shadowing property is preserved by a topological conjugacy and that for a continuous map $f$ on a compact metric space and any positive integer $n, f$ has the asymptotically shadowing property if and only if $f^{n}$ has the asymptotically shadowing property. As an equivalent definition, we have

Proposition 1.2. $f$ has the asymptotically shadowing property if and only if for every $\varepsilon>0$ there exist a real number $\delta>0$ and an integer $l \geq 0$ such that for any $\delta$-pseudo-orbit $\left\{x_{0}, \ldots, x_{n}, \ldots\right\}$ of $f,\left\{f^{l}\left(x_{0}\right), \ldots, f^{l}\left(x_{n}\right), \ldots\right\}$ is $\varepsilon$-shadowed under $f$.

The main result of this paper is

Theorem 1.3. If $f: X \rightarrow X$ is continuous and onto, then the following are equivalent

(1) the shadowing property of $f$ on $(X, d)$,

(2) the shadowing property of $\sigma_{f}$ on $\langle X ; f ; \underline{d}\rangle$,

(3) the asymptotically shadowing property of $f$ on $(X, d)$,

(4) the asymptotically shadowing property of $\sigma_{f}$ on $\langle X ; f ; \underline{d}\rangle$.

In $\S 2$ we will prove Proposition 1.2. In $\S 3$ we will prove Theorem 1.3, and in $\S 4$ we will use Theorem 1.3 to show the shift map of the pseudoarc that was constructed by Henderson [12] has the shadowing property.

\section{The PROOF OF Proposition 1.2}

Lemma 2.1. Suppose $(X, d)$ is a compact metric space and $f: X \rightarrow X$ is continuous. Given $\varepsilon>0$ and $M>0$, there is $\delta>0$ such that every $\delta$-pseudo-orbit $\left\{x_{0}, \ldots, x_{n}, \ldots\right\}$ satisfies

$$
d\left(f^{M}\left(x_{k}\right), x_{M+k}\right) \leq \varepsilon \quad \text { for all } k \geq 0 .
$$

Proof. By the uniform continuity of $f$ and

$$
d\left(f^{M}\left(x_{k}\right), x_{M+k}\right) \leq \sum_{j=0}^{M-1} d\left(f^{M-j}\left(x_{k+j}\right), f^{M-j-1}\left(x_{k+j+1}\right)\right) \quad \text { for all } k \geq 0 .
$$

Proof of Proposition 1.2. $(\Leftarrow)$ Given $\varepsilon>0$, there exist $\tau>0$ and $l>0$ such that for every $\tau$-pseudo-orbit $\left\{x_{0}, \ldots, x_{n}, \ldots\right\}$ there exists $z \in X$ that $(\varepsilon / 2)$ shadows $\left\{x_{l}, x_{l+1}, \ldots\right\}$. By Lemma 2.1 , choose $\delta \in(0, \tau)$ small enough that $d\left(f^{l}\left(x_{k}\right), x_{l+k}\right) \leq \varepsilon / 2$ for $k=0,1, \ldots$ If $\left\{x_{0}, \ldots, x_{n}, \ldots\right\}$ is a $\delta$-pseudoorbit and $z$ is as in the above, then

$$
d\left(f^{l}\left(x_{k}\right), f^{k}(z)\right) \leq d\left(f^{l}\left(x_{k}\right), x_{l+k}\right)+d\left(x_{l+k}, f^{k}(z)\right) \leq \varepsilon .
$$


$(\Rightarrow)$ Given $\varepsilon>0$, there exist $\tau>0$ and $N>0$ such that for every $\delta$ pseudo-orbit $\left\{x_{0}, \ldots, x_{n}, \ldots\right\}$ we can find $z \in X$ such that

$$
d\left(f^{N}\left(x_{k}\right), f^{k}(z)\right) \leq \varepsilon / 2 \text { for all } k \geq 0,
$$

and, by Lemma 2.1 , such a $\delta$ can be chosen small enough that

$$
d\left(f^{N}\left(x_{k}\right), x_{N+k}\right) \leq \varepsilon / 2 \text { for all } k \geq 0 .
$$

Thus, $d\left(f^{k}(z), x_{N+k}\right) \leq d\left(f^{k}(z), f^{N}\left(x_{k}\right)\right)+d\left(f^{N}\left(x_{k}\right), x_{N+k}\right) \leq \varepsilon$ for all $k \geq$ 0 .

\section{THE PROOF OF THEOREM 1.3}

Let $M$ be the diameter of $X$. If $f$ is onto, for $x_{0}$ there are preimages $x_{1}, x_{2}, \ldots$ satisfying $f\left(x_{i+1}\right)=x_{i}$ for all $i \geq 0$. Then $\left(x_{0}, x_{1}, \ldots\right) \in\langle X ; f\rangle$ by definition. In this case we will use the notation $\left(x_{0}, *\right)$ to denote the element $\left(x_{0}, x_{1}, \ldots\right)$ of $\langle X ; f\rangle$.

Proof that $(3) \Rightarrow(4)$. For any $\varepsilon>0$, choose $m \geq \log (2 M / \varepsilon) / \log 2$, then

$$
1 / 2^{m} \leq \varepsilon / 2 M \text {. }
$$

By the uniform continuity of $f$ on $X$, there is $\tau>0$ such that

$$
d(x, y) \leq \tau \Rightarrow d\left(f^{i}(x), f^{i}(y)\right) \leq \varepsilon / 4 \quad \text { for } 0 \leq i \leq m,
$$

and then by the shadowing property of $f$ on $X$, we can find $\delta>0$ and $l>0$ so that if $\left\{x_{0}, \ldots, x_{n}, \ldots\right\}$ is a $\delta$-pseudo-orbit of $f$ then $\left\{f^{l}\left(x_{0}\right), \ldots\right.$, $\left.f^{l}\left(x_{n}\right), \ldots\right\}$ is $\tau$-shadowed.

Suppose $\{\underline{y}(0), \ldots, \underline{y}(n), \ldots\}$ is a $\left(\delta / 2^{m}\right)$-pseudo-orbit of $\sigma_{f}$ in $\langle X ; f ; \underline{d}\rangle$; that is,

$$
\underline{d}\left(\underline{y}(n+1), \sigma_{f}[\underline{y}(n)]\right) \leq \delta / 2^{m} \text { for all } n \geq 0 .
$$

Illustratively we write $\underline{y}(n)=\left(y_{0}^{n}, y_{1}^{n}, \ldots\right)$, then from (3) we get

$$
\frac{d\left(y_{m}^{n+1}, y_{m-1}^{n}\right)}{2^{m}} \leq \underline{d}\left(\underline{y}(n+1), \sigma_{f}[\underline{y}(n)]\right) \leq \frac{\delta}{2^{m}}
$$

or

$$
d\left(y_{m}^{n+1}, f\left(y_{m}^{n}\right)\right)=d\left(y_{m}^{n+1}, y_{m-1}^{n}\right) \leq \delta .
$$

Thus, $\left\{y_{m}^{0}, \ldots, y_{m}^{n}, \ldots\right\}$ is a $\delta$-pseudo-orbit of $f$. By the choice of $\delta$ and the shadowing property of $f$, there exists $x_{0} \in X$ that $\tau$-shadows $\left\{f^{l}\left(y_{m}^{0}\right), \ldots\right.$, $\left.f^{l}\left(y_{m}^{n}\right), \ldots\right\}$; that is,

$$
d\left(f^{n}\left(x_{0}\right), f^{l}\left(y_{m}^{n}\right)\right) \leq \tau \quad \text { for any } n \geq 0 .
$$

It follows from (2) that

$$
d\left(f^{i}\left[f^{n}\left(x_{0}\right)\right], f^{i}\left[f^{l}\left(y_{m}^{n}\right)\right]\right) \leq \varepsilon / 4 \quad \text { for } 0 \leq i \leq m .
$$

Now letting $\underline{z}=\left(f^{m}\left(x_{0}\right), \ldots, f\left(x_{0}\right), x_{0}, *\right) \in\langle X, f\rangle$, we have

$$
\sigma_{f}^{n}(\underline{z})=\left(f^{n+m}\left(x_{0}\right), \ldots, f\left(x_{0}\right), x_{0}, *\right) .
$$


Furthermore, for every $n \geq 0$

$$
\begin{aligned}
\underline{d}\left(\sigma_{f}^{l}\right. & {\left.[\underline{y}(n)], \sigma_{f}^{n}(\underline{z})\right) } \\
& \leq \sum_{i=0}^{m} \frac{d\left(f^{l}\left(y_{i}^{n}\right), f^{n+m-i}\left(x_{0}\right)\right)}{2^{i}}+\frac{\varepsilon}{2} \quad[\text { by }(1)] \\
& =\sum_{i=0}^{m} \frac{d\left(f^{l}\left(y_{m-i}^{n}\right), f^{n+i}\left(x_{0}\right)\right)}{2^{m-i}}+\frac{\varepsilon}{2} \\
& =\sum_{i=0}^{m} \frac{d\left(f^{i}\left[f^{l}\left(y_{m}^{n}\right)\right], f^{i}\left[f^{n}\left(x_{0}\right)\right]\right)}{2^{m-i}}+\frac{\varepsilon}{2} \quad\left[\text { since } f^{i}\left(y_{m}^{n}\right)=y_{m-i}^{n}\right] \\
& \leq \frac{\varepsilon}{4} \sum_{i=0}^{m} \frac{1}{2^{m-i}}+\frac{\varepsilon}{2} \leq \frac{\varepsilon}{2}+\frac{\varepsilon}{2}=\varepsilon .
\end{aligned}
$$

This implies that $\underline{z} \varepsilon$-shadows $\left\{\sigma_{f}^{l}[\underline{y}(0)], \ldots, \sigma_{f}^{l}[\underline{y}(n)], \ldots\right\}$ under $\sigma_{f}$.

Proof that $(4) \Rightarrow(3)$. For a given $\varepsilon>0$, by the asymptotically shadowing property of $\sigma_{f}$, there exist $\tau>0$ and $l>0$ such that if $\{\underline{y}(0), \ldots, \underline{y}(n), \ldots\}$ is a $\tau$-pseudo-orbit of $\sigma_{f}$, then

$$
\left\{\sigma_{f}^{l}[\underline{y}(0)], \ldots, \sigma_{f}^{l}[\underline{y}(n)], \ldots\right\}
$$

is $\varepsilon$-shadowed. Choose $p \geq \max \{\log (2 M / \tau) / \log 2,0\}$, then

$$
M / 2^{p} \leq \tau / 2
$$

By the uniform continuity of $f$ on $X$, choose $\delta>0$ small enough so that every $\delta$-pseudo-orbit $\left\{x_{n}\right\}$ of $f$ satisfies

$$
\sum_{i=0}^{p} \frac{d\left(f^{i}\left(x_{n+1}\right), f^{i+1}\left(x_{n}\right)\right)}{2^{p-i}} \leq \frac{\tau}{2} .
$$

Define $\underline{y}(n)=\left(f^{p}\left(x_{n}\right), \ldots, x_{n}, *\right) \in\langle X ; f\rangle$ for $n=0,1, \ldots$ Then

$$
\begin{aligned}
\underline{d}\left(\underline{y}(n+1), \sigma_{f}[\underline{y}(n)]\right) & \leq \sum_{i=0}^{p} \frac{d\left(f^{i}\left(x_{n+1}\right), f^{i+1}\left(x_{n}\right)\right)}{2^{p-i}}+\frac{\tau}{2} \quad[\text { by (7)] } \\
& \leq \frac{\tau}{2}+\frac{\tau}{2}=\tau
\end{aligned}
$$

that is, $\{\underline{y}(0), \ldots, \underline{y}(n), \ldots\}$ is a $\tau$-pseudo-orbit of $\sigma_{f}$.

By the choice of $\bar{\tau}$, there exists $\underline{z}=\left(z_{0}, z_{1}, \ldots\right) \in\langle X ; f\rangle$ that $\varepsilon$-shadows

$$
\left\{\sigma_{f}^{l}[\underline{y}(0)], \ldots, \sigma_{f}^{l}[\underline{y}(n)], \ldots\right\}
$$

that is

$$
\underline{d}\left(\sigma_{f}^{l}[\underline{y}(n)], \sigma_{f}^{n}(\underline{z})\right) \leq \varepsilon \quad \text { for } n=0,1,2, \ldots .
$$

Notice that $\sigma_{f}^{n}(\underline{z})=\left(f^{n}\left(z_{0}\right), f^{n-1}\left(z_{0}\right), \ldots\right)$; we have

$$
d\left(f^{l+p}\left(x_{n}\right), f^{n}\left(z_{0}\right)\right) \leq \underline{d}\left(\sigma_{f}^{l}[\underline{y}(n)], \sigma_{f}^{n}(\underline{z})\right) \leq \varepsilon \quad \text { for } n=0,1,2, \ldots
$$

It follows that $\left\{f^{l+p}\left(x_{0}\right), \ldots, f^{l+p}\left(x_{n}\right), \ldots\right\}$ is $\varepsilon$-shadowed under $f$ by $z_{0}$. 
Proof that $(1) \Rightarrow(2)$ and $(2) \Rightarrow(3)$. The proof that $(1) \Rightarrow(2)$ is exactly a copy of the proof that $(3) \Rightarrow(4)$ by taking $l=0$ and the proof that $(2) \Rightarrow(3)$ is exactly a copy of the proof that $(4) \Rightarrow(3)$ by taking $l=0$.

Proof that $(3) \Rightarrow(1)$. Give $\varepsilon>0$, let $\delta>0$ and $N \geq 0$ be the numbers as in Definition 1.1. Suppose $\left\{x_{0}, \ldots, x_{n}, \ldots\right\}$ is any $\delta$-pseudo-orbit of $f$. Since $f$ is onto, there exists $z \in X$ such that $f^{N}(z)=x_{0}$. Thus, $\left\{z, f(z), \ldots, f^{N-1}(z), x_{0}, \ldots, x_{n}, \ldots\right\}$ is also a $\delta$-pseudo-orbit which is $\varepsilon$ shadowed from the term $x_{0}$, according to the asymtotically shadowing property of $f$.

\section{THE SHADOWING PROPERTY FOR PSEUDOARCS}

A direct application of Theorem 1.3 is to show that the shift map of some pseudoarc has the shadowing property.

Let $X$ be a continuum. We say that $X$ is chainable if for any $\varepsilon>0$ there are finitely many open sets whose diameters are smaller than $\varepsilon$, say $\mathscr{O}_{1}, \mathscr{O}_{2}, \ldots, \mathscr{O}_{n}$, which covers $X$ and $\mathscr{O}_{i} \cap \mathscr{O}_{j} \neq \varnothing$ iff $|i-j| \leq 1 . X$ is also called snakelike. We say that $X$ is decomposable if $X$ can be written as the union of two proper subcontinua. If $X$ is not decomposable, the $X$ is indecomposable. $X$ is hereditarily indecomposable if each of its subcontinua is indecomposable. A pseudoarc is a continuum that is homeomorphic to a hereditarily incomposable chainable continuum. It is known that the inverse limit spaces of the maps on the interval are chainable. The inverse limit space of some maps on the interval can be a pseudoarc.

In this section, we consider the map on $[0,1]$ constructed by Henderson [12] to exhibit a pseudoarc as its inverse limit space. According to [12], this map $f:[0,1] \rightarrow[0,1]$ has the following properties:

(1) $f$ is a continuous function from $[0,1]$ onto itself;

(2) $f(x) \leq x$ for all $x \in[0,1]$ and $f$ has only two fixed points at the ends of $[0,1]$;

(3) the inverse limit space of $f$ is a pseudoarc.

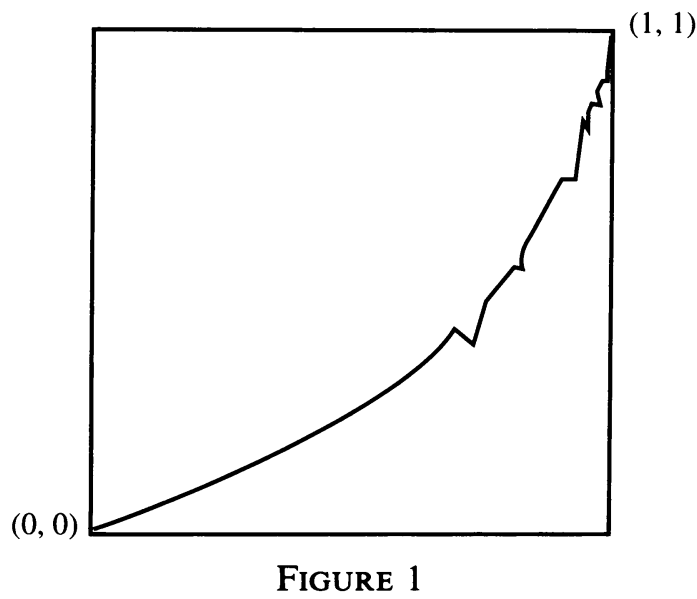

By Theorem 1.3, to show the shadowing property for the shift map on the pseudoarc, we need only to show that $f$ has the shadowing property. This is 
true because we have a result as follows:

Lemma 4.1. If $f:[0,1] \rightarrow[0,1]$ is continuous and has fixed points only at the ends of the interval, then $f$ has the shadowing property.

Proof. Without loss of generality, we assume that $f(x)<x$ for all $x \in(0,1)$ and both 0 and 1 are fixed by $f$.

Given $\varepsilon \in\left(0, \frac{1}{3}\right)$, let $U_{\varepsilon}=[0, \varepsilon), V_{\varepsilon}=(1-\varepsilon, 1]$, and

$$
\gamma=\min _{\varepsilon / 2 \leq x \leq 1-\varepsilon}\{|x-f(x)|\} \text {. }
$$

Thus $\gamma>0$ and $f(x) \leq x-\gamma$ for all $x \in[\varepsilon / 2,1-\varepsilon]$. If also $f(x) \in[\varepsilon / 2,1-\varepsilon]$ then $f^{2}(x) \leq f(x)-\gamma \leq x-2 \gamma$. Let $N \geq(2-3 \varepsilon) / 2 \gamma$, we have $f^{n}(x) \in U_{\varepsilon / 2}$ for all $n \geq N$ and all $x \in[0,1-\varepsilon]$.

By Lemma 2.1, there is $\tau>0$ such that if $\left\{z_{0}, \ldots, z_{n}, \ldots\right\}$ is a $\tau$-pseudoorbit of $f$ with $z_{0} \in[0,1-\varepsilon]$ then $\left|z_{N}-f^{N}\left(z_{0}\right)\right| \leq \varepsilon / 2$, moreover, $z_{N} \in U_{\varepsilon}$. By the uniform continuity of $f$, there exists $\sigma>0$ such that every $\sigma$-pseudoorbit $\left\{z_{0}, \ldots, z_{n}, \ldots\right\}$ satisfies $\left|f^{j}\left(z_{0}\right)-z_{j}\right| \leq \varepsilon / 2$ for every $0 \leq j \leq N$ and there exists $\eta>0$ such that if $|x-y| \leq \eta$ then $\left|f^{j}(x)-f^{j}(y)\right| \leq \varepsilon / 2$ for $0 \leq j \leq N$.

Choose $\delta \in(0, \min \{\tau, \sigma, \gamma, \eta,(1-3 \varepsilon) / 2\})$; let $\left\{x_{0}, \ldots, x_{n}, \ldots\right\}$ be a $\delta$-pseudo-orbit in $[0,1]$. If $x_{0} \in[\varepsilon / 2,1-\varepsilon]$ then

$$
x_{\mathrm{i}}=f\left(x_{0}\right)+x_{1}-f\left(x_{0}\right) \leq x_{0}-(\gamma-\delta) \leq x_{0} ;
$$

that is, $x_{1} \in[0,1-\varepsilon]$. It is clear that if $x_{0}[0, \varepsilon / 2)$ we also have $x_{1} \in[0,1-\varepsilon]$. By induction, if $x_{0} \in[0,1-\varepsilon]$ then $x_{n} \in[0,1-\varepsilon]$ for all $n \geq 0$ and, by the choice of $N, x_{n} \in U_{\varepsilon}$ for any $n \geq N$. By the choice of $\sigma$, if $x_{0} \in[0,1-\varepsilon]$ then $x_{0} \quad \varepsilon$-shadows $\left\{x_{0}, \ldots, x_{n}, \ldots\right\}$.

Now if $x_{0} \in V_{\varepsilon}$, we have two cases:

Case 1. For all $n \geq 0, x_{n} \in V_{\varepsilon}$. Then $y=1 \varepsilon$-shadows $\left\{x_{0}, \ldots, x_{n}, \ldots\right\}$;

Case 2. There is $x_{k} \in[0,1-\varepsilon]$. Let $k_{0}$ be the largest $k$ such that $x_{k} \in$ $V_{\varepsilon}$. Thus, by the choice of $\delta, x_{n} \in[0,1-\varepsilon]$ for all $n>k_{0}$ and $\left\{x_{k_{0}+1}\right.$, $\left.x_{k_{0}+2}, \ldots\right\}$ is $(\varepsilon / 2)$-shadowed by $x_{k_{0}+1}$. Notice that $f([1-\varepsilon, 1]) \supset[1-\varepsilon, 1]$, so $f^{k_{0}}([1-\varepsilon, 1]) \supset[1-\varepsilon, 1]$. Therefore, there is $y \in[1-\varepsilon, 1] \cap \cdots \cap f^{-k_{0}}([1-$ $\varepsilon, 1])$ such that $f^{k_{0}}(y)=x_{k_{0}}$. Then, $f^{j}(y), x_{j} \in V_{\varepsilon}$ for $j=0, \ldots, k_{0}$. Furthermore, $\left|f^{k}(y)-x_{k}\right|<\varepsilon$ for every $k=0, \ldots, k_{0}$. For $j=1, \ldots, N$,

$$
\begin{aligned}
\left|f^{k_{0}+j}(y)-x_{k_{0}+j}\right| & \leq\left|f^{j}\left(x_{k_{0}}\right)-f^{j-1}\left(x_{k_{0}+1}\right)\right|+\left|f^{j-1}\left(x_{k_{0}+1}\right)-x_{k_{0}+j}\right| \\
& \leq \frac{\varepsilon}{2}+\frac{\varepsilon}{2} \leq \varepsilon .
\end{aligned}
$$

For $j \geq k_{0}+N, f^{j}(y), x_{j} \in U_{\varepsilon}$. Therefore, $y \varepsilon$-shadows $\left\{x_{0}, \ldots\right.$, $\left.x_{n}, \ldots\right\}$.

Example 4.2. Let $f_{c}:[0,1] \rightarrow[0,1]$ be a continuous monotone map with the property: $f(x)>x$ if and only if $x \in[0, c) \cup(c, 1)$ for some $c \in(0,1)$.

Notice that $f_{c}$ does not have the shadowing property since for any $\delta>0$ there exists a $\delta$-pseudo-orbit from 0 to 1 , but there is no true orbit crossing through $c$ to the other side.

We show that $f_{c}$ has the asymptotically shadowing property.

Given $\varepsilon>0$ with $4 \varepsilon<1-c$, by assumption there exists $N>0$ such that

$$
f_{c}^{n}([0,1]) \subseteq(c-\varepsilon / 4,1] \text { for all } n \geq N \text {. }
$$




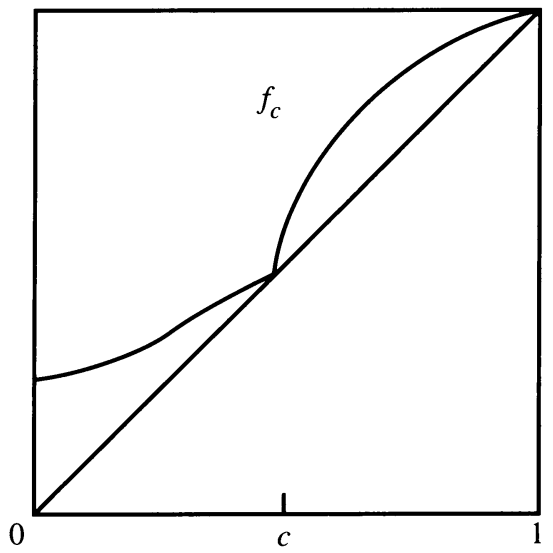

FIGURE 2

Then by Lemma 2.1 , there exists $\delta_{1}>0$ such that every $\delta_{1}$-psuedo-orbit $\left\{x_{0}, \ldots, x_{n}, \ldots\right\}$ satisfies that

$$
f_{c}^{N}\left(x_{n}\right) \in(c-\varepsilon / 4,1] \text { for all } n \geq 0 .
$$

Let $\gamma=f(c+\varepsilon / 4)-(c+\varepsilon / 4)$; then any $\gamma$-pseudo-orbit starting from a point in $[c+\varepsilon / 4,1]$ is totally contained in $[c+\varepsilon / 4,1]$.

By Lemma 4.1, $\left.f_{c}\right|_{[c, 1]}$ has the shadowing property; thus there exists $\delta_{2} \in$ $(0, \gamma)$ such that every $\delta_{2}$-pseudo-orbit in $[c, 1]$ is $(\varepsilon / 2)$-shadowed. By the uniform continuity of $f_{c}$, there exists $\delta \in\left(0, \delta_{1}\right)$ such that

$$
|x-y| \leq \delta \Rightarrow\left|f_{c}^{N}(x)-f_{c}^{N}(y)\right| \leq \delta_{2} .
$$

Now we show that if $\left\{y_{0}, \ldots, y_{n}, \ldots\right\}$ is a $\delta_{2}$-pseudo-orbit in $[c-\varepsilon / 4,1]$, then there exists a $\delta_{2}$-pseudo-orbit $\left\{z_{0}, \ldots, z_{n}, \ldots\right\}$ in $[c, 1]$ that is $(\varepsilon / 2)$ close to $\left\{y_{0}, \ldots, y_{n}, \ldots\right\}$. In fact, if $y_{n} \in(c-\varepsilon / 4, c+\varepsilon / 4)$ for all $n \geq 0$, let $z_{n}=c$ for all $n \geq 0$; if there exists $y_{n} \in[c+\varepsilon / 4,1]$ and we let $n_{0}$ be the smallest index for which $y_{n_{0}} \in[c+\varepsilon / 4,1]$, then we have a $\delta_{2}$-pseudo-orbit $\left\{z_{0}, \ldots, z_{n}, \ldots\right\}$ in $[0,1]$ defined by

$$
z_{n}= \begin{cases}f_{c}^{-n_{0}+n}\left(y_{n_{0}}\right) & \text { for } 0 \leq n \leq n_{0} \\ y_{n} & \text { for } n>n_{0}\end{cases}
$$

If $\left\{x_{0}, \ldots, x_{n}, \ldots\right\}$ is a $\delta$-pseudo-orbit in $[0,1]$, then $\left\{f_{c}^{N}\left(x_{0}\right), \ldots\right.$, $\left.f_{c}^{N}\left(x_{n}\right), \ldots\right\}$ is a $\delta_{2}$-pseudo-orbit in $[c-\varepsilon / 4,1]$ that is $\varepsilon$-close to a $\delta_{2}$-pseudoorbit $\left\{z_{0}, \ldots, z_{n}, \ldots\right\}$ in $[c, 1]$. Thus there exists a true orbit $(\varepsilon / 2)$-shadows $\left\{z_{0}, \ldots, z_{n}, \ldots\right\}$ and it also $\varepsilon$-shadows $\left\{f_{c}^{N}\left(x_{0}\right), \ldots, f_{c}^{N}\left(x_{n}\right), \ldots\right\}$.

\section{ACKNOWLEDGMENT}

We would like to thank the referee for the recommendation and some suggestions. 


\section{REFERENCES}

1. D. V. Anosov, On a class of invariant sets for smooth dynamical systems, Proc. Fifth Internat. Conf. on Nonlinear Oscillations, vol. 2, Math. Inst. Ukrainian Acad. Sci., Kiev, 1970, pp. 39-45. (Russian)

2. M. Barge and R. Swanson, Rotation shadowing properties of circle and annulus maps, Ergodic Theory Dynamical Systems 8 (1988), 509-521.

3. __ Pseudo-orbits and topological entropy, Proc. Amer. Math. Soc., Vol. 109, No. 2 (1990), 559-566.

4. M. L. Blank, Metric properties of $\varepsilon$-trajectories of dynamical systems with stochastic behaviour, Ergodic Theory Dynamical Systems 8 (1988), 365-378.

5. R. Bowen, On axiom A diffeomorphisms, CBMS Reg. Conf. Ser. Math., vol. 35, Amer. Math. Soc., Providence, RI, 1978.

6. A. Boyarski and P. Góra, The pseudo-orbit shadowing property for Markov operators in the space of probability density functions, preprint, October 1989.

7. L. Chen, Linking and the shadowing property for piecewise monotone maps, Proc. Amer. Math. Soc. Vol. 113, No. 1 (1991), 251-263.

8. $\ldots$, On the shadowing property for nondegenerated zero entropy piecewise monotone maps, SUNY Stony Brook preprint series, \#1990/9, 1990.

9. E. M. Coven, I. Kan, and J. A. Yorke, Pseudo-orbit shadowing in the family of tent maps, Trans. Amer. Math. Soc. 308 (1988), 227-241.

10. J. E. Franke and J. F. Selgrade, Hyperbolicity and chain recurrence, J. Differential Equations 26 (1977), 27-36.

11. T. Gedeon and M. Kuchta, Shadowing property of continuous maps, preprint.

12. G. W. Henderson, The pseudo-arc as an inverse limit with one binding map, Duke Math. J. 31 (1964), 421-425.

13. I. Kan, Shadowing properties of quadratic maps, preprint.

14. M. Komuro, The pseudo orbit tracing properties on the space of probability measures, Tokyo J. Math. 7 (1984), 461-468.

15. S. H. Li, The dynamical properties of the shift maps on the inverse limit space, to appear in Ergod. Th. \& Dynam. Sys.

16. P. Walters, On the pseudo-orbit tracing property and its relationship to stability, Lecture Notes in Math., vol. 668, Springer-Verlag, Berlin, Heidelbery, pp. 191-210.

17. R. F. Williams, One-dimensional nonwandering sets, Topology 6 (1967), 473-487.

Department of Mathematics Sciences, Memphis State University, Memphis, Tennessee 38152

E-mail address: lchen@jade.tufts.edu

Department of Mathematics, University of Florida, Gainesville, Florida 32601-2082

E-mail address: shi@math.ufl.edu

Current address: (Liang Chen) 91 Perkins Street, Somerville, Massachusetts 02145 